\title{
Solar-driven gas turbine power plants
}

\author{
A. Medina ${ }^{1}$, S. Sánchez-Orgaz ${ }^{2}$, A. Calvo Hernández ${ }^{3}$ \\ ${ }^{1}$ Departamento de Física Aplicada, \\ Universidad de Salamanca, \\ 37008 Salamanca, Spain \\ Phone number:+0034 923294 436, e-mail: amd385@usal.es \\ ${ }^{2}$ Departamento de Física, Ingeniería y Radiología Médica \\ ETSII de Béjar, Universidad de Salamanca, \\ 37700 Béjar, Salamanca, Spain \\ Phone number:+0034 923408 080, e-mail: susan@usal.es \\ ${ }^{3}$ Departamento de Física Aplicada and IUFFYM \\ Universidad de Salamanca, \\ 37008 Salamanca, Spain \\ Phone number:+0034 923294 436, e-mail: anca@usal.es
}

January 9, 2013

\begin{abstract}
A general model for an irreversible solar-driven Brayton multi-step heat engine is presented. The model incorporates a regenerator, an arbitrary number of turbines $\left(N_{t}\right)$ and compressors $\left(N_{c}\right)$ and the corresponding reheating and intercooling processes; thus the solar-driven Ericsson cycle is a particular case where $N_{t}, N_{c} \rightarrow \infty$. For the solar collector we assume linear heat losses and for the Brayton multi-step cycle irreversibilities arising from the non-ideal behavior of turbines and compressors, pressure drops in the heat input and heat release, heat leakage through the plant to the surroundings, and non-ideal couplings of the working fluid with the external heat reservoirs. We obtain the collector temperatures at which maximum overall efficiency $\eta_{\max }$ is reached as a function of the thermal plant pressure ratio and a detailed comparison for several plant configurations is given.
\end{abstract}

\section{Key Words}

Thermodynamic optimization, Solar-driven heat engine, Multi-step gas-turbine, Irreversibilities, Cy- cle performance.

\section{Introduction}

Because of saving energy and minimizing environmental impact strategies, solar-driven heat engines are attracting much interest nowadays and, as a consequence, different heat engine cycle models coupled to a solar collector have been investigated. Thermodynamic studies analyzing different sources of irreversibilities and different optimization criteria have been reported for solar-driven Carnot, Ericsson, Stirling, and Braysson cycles.

In particular, steam, gas or combined turbine cycles are realistic examples to generate electricity when the heat source is solar energy. Compared to conventional steam turbines, gas turbines have relatively lower thermal efficiencies but bear the advantage of their compact building and low construction costs. Moreover, gas turbines can be operated very dynamically (quick start-up) and at significantly lower pressures. The needed heat input can be supplied at least partially (hybrid systems) by concentrating solar collectors using tower-plant or 
dish/engine technology. The turbine exhaust energy could be used in a thermal recuperation process through a bottoming cycle.

In recent years several prototypes and experimental facilities of solar-driven gas turbine power plants have been developed $[1,2,3,4]$. They usually work on an hybrid solar/fossil fuel operation, so that an standard combustion chamber can compensate the intermittent nature of solar irradiance. The future commercial interest of this alternative for electric power generation relies on the reduction of investment and generating costs and on an increase of the plant thermal efficiency. Theoretical and computer analyses on the effect of the main irreversibility sources over the overall thermal efficiency and the optimal values of some basic thermodynamic parameters are necessary steps in order to design efficient solar-driven thermal plants.

Recently, we have developed a theoretical model [5] for a regenerative multi-step Brayton heat engine (with standard heat input through a combustion chamber) with an arbitrary number of turbines $N_{t}$ and compressors $N_{c}$ and incorporating all the irreversibility sources existing in a real plant: the non-ideal behavior of turbines and compressors, pressure drops in the heat input and heat release, regenerator irreversibility, heat leakage through the plant to the surroundings, and non-ideal couplings of the working fluid with the external heat reservoirs. In the present work we combine the Brayton heat engine model previously developed [5] with a solar collector in such a way that the heat input completely comes from the solar collector. Such solarized multistep Brayton system offers optimization possibilities completely different that those of its components separately. The analysis of this combined system and its optimization is the main issue of our work.

We shall see that the incorporation of a reasonable number of compression-expansion stages and the consideration of optimum pressure and temperature ratios greatly improves the performance of this kind of solar-driven thermal power plants with respect to the simple one-step cycle. This increase reaches a $65 \%$ for an arrangement with two compressors and two turbines. Our model could be considered as an initial global simulation scheme in order to optimize the overall performance records of the plant in terms of a reduced set of parameters (easy to estimate) arising from the irreversibility sources affecting this kind of systems. One of its main advantages is the flexibility since it can be applied to several plant arrangements, independently of the considered number of compressors and turbines and of the details of the solar collector.

\section{Theoretical model}

The configuration scheme and the $T-S$ diagram of our model for an irreversible solar-driven multistep Brayton heat engine are depicted in Fig. 1. The Brayton heat engine absorbs a net heat rate $\left|\dot{Q}_{H}\right|$ from the solar collector at temperature $T_{H}$ and releases a net heat rate $\left|\dot{Q}_{L}\right|$ to the ambient at temperature $T_{L}$. We also assume a linear heat leakage $\left|\dot{Q}_{H L}\right|$ directly from the hot reservoir at $T_{H}$ to the cold heat sink at $T_{L}[6]$.

Following $[7,8]$ we consider a concentrating collector for which heat losses at low and intermediate temperatures are essentially associated to conduction and convection while at high enough temperatures radiation losses are dominant. In this model the useful energy delivered to the heat engine, $\left|\dot{Q}_{H}\right|$, and the efficiency of the solar collector $\eta_{s}$ can be written, respectively, as

$$
\begin{gathered}
\left|\dot{Q}_{H}\right|=\eta_{0} G A_{a}-U_{L} A_{r} T_{L}(\tau-1), \\
\eta_{s}=\frac{\left|\dot{Q}_{H}\right|}{G A_{a}}=\eta_{0}[1-(\tau-1) M]
\end{gathered}
$$

In these equations $\tau=T_{H} / T_{L}$ denotes the heat reservoirs temperature ratio, $G$ is the solar irradiance, $A_{a}$ and $A_{r}$ are, respectively, the aperture and absorber areas, $C=A_{a} / A_{r}$ is the concentration ratio, $\eta_{0}$ is the effective transmittance-absorptance product (optical efficiency), and $M$ is defined as $M=U_{L} T_{L} /\left(\eta_{0} G C\right)$, where $U_{L}$ is an overall effective coefficient accounting for radiation losses in a linearized heat loss term $[7,8]$. We have checked that all the results in this work are not significantly affected by the consideration of explicit radiation losses depending on $\tau^{4}[9]$. The shape of all the figures we shall show remains unaltered, and there are only very small fluctuations on some particular numerical values.

The basic mathematical equations of the model can be found in $[5,9,10]$. Equations for heat input and heat release, allow to obtain the efficiency of the Brayton heat engine, $\eta_{h}=|\dot{W}| /\left|\dot{Q}_{H}\right|=$ 


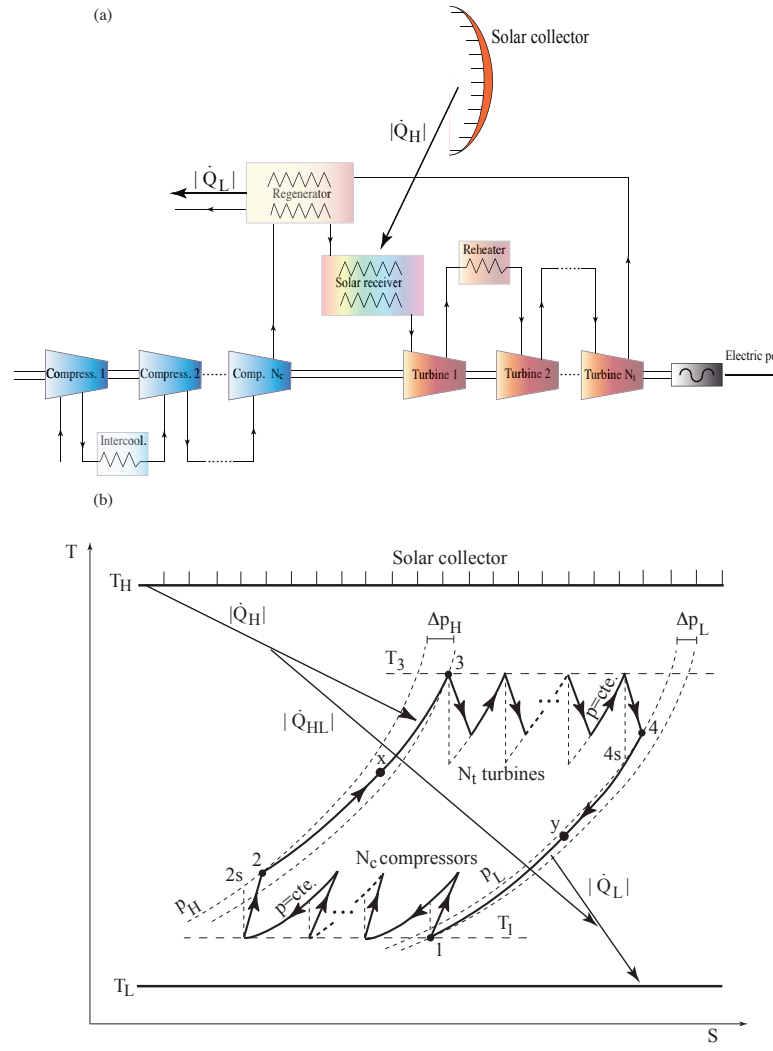

Figure 1: (a) Schematic representation of a solar powered multistage gas turbine plant and (b) $T-S$ diagram of our model for a solar-driven multi-step regenerative Brayton cycle.

$1-\left|\dot{Q}_{L}\right| /\left|\dot{Q}_{H}\right|$, where $|\dot{W}|$ is the net power output of the cycle. This thermal efficiency emerges as a function of the geometrical parameters that characterize the shape and size of the cycle the temperature, $\tau=T_{H} / T_{L}$, and pressure ratios, $\left(r_{p}\right)$, the number of turbines $\left(N_{t}\right)$ and compressors $\left(N_{c}\right)$, the turbine and compressor efficiencies $\left(\epsilon_{t}, \epsilon_{c}\right)$, the pressure losses $\left(\rho_{H}, \rho_{L}\right)$, the regenerator efficiency $\left(\epsilon_{r}\right)$, the heat-leak losses $(\xi)$, and of the parameters accounting for the external irreversibilities $\left(\epsilon_{H}, \epsilon_{L}\right)$.

This model has been validated and compared with experimental facilities in [11]. Particularly, the predictions of the model for the commercial one-turbine one-compressor regenerative plant Turbec T100 (ABB/Volvo) [12, 13] differ $2.7 \%$ for efficiency, $6.7 \%$ for the power output, and $4.2 \%$ for the heat input. Predicted outputs were also compared with the experimental ones for a re- generative plant with two-compressors and oneturbine $[14,15]$. In this case the model overestimates experimental efficiency by $2.8 \%$ and power output by $1.1 \%$. These results show a fair agreement of our Brayton multi-step theoretical model predictions with real plants.

The efficiency of the overall solar-driven plant $\eta=|\dot{W}| / G A_{a}$ can be expressed as the product of the efficiencies of the solar collector and of the multi-step Brayton heat engine:

$$
\eta=\frac{|\dot{W}|}{G A_{a}}=\frac{|\dot{W}|}{\left|\dot{Q}_{H}\right|} \frac{\left|\dot{Q}_{H}\right|}{G A_{a}} \equiv \eta_{h} \eta_{s}
$$

\section{Optimization with respect to the temperature ratio}

The goal of this section is to present a numerical analysis accounting for all the main irreversibilities affecting the real processes in a solar powered gas turbine power plant.

We show in Fig. 2 the efficiency as a function of $\tau$ for the basic B configuration (one compressor and one turbine) and some limit cases: E (infinite compressors and turbines), IT (infinite turbines and one compressor), and IC (infinite compressors and one turbine). We take as reference for the irreversibility parameters the following values: $\epsilon_{t}=0.89$, $\epsilon_{c}=0.84[16,13,17], \epsilon_{r}=0.85[12,16,17]$, and $\epsilon_{H}=\epsilon_{L} \equiv \epsilon=0.90[16], \rho_{H}=\rho_{L}=0.98, \xi=0.02$, $T_{L}=300 K$, and $\gamma=1.4$ [5]. Parameters for the solar collector are: $\eta_{0}=0.84$ and $M=0.29$ [10]. From this figure we mention three relevant facts. First, Ericsson efficiency is always above IC and IT, and these are in turn over the simple regenerative B-configuration. This is valid for any value of $r_{p}$ and $\tau$. Second, the intervals for $\tau$ giving positive efficiencies become narrower for B, IT, and IC configurations as $r_{p}$ increases. Third, for the $r_{p}$-values presented $\left(r_{p}=5,15,20\right)$, the maximum efficiency for each configuration decreases as $r_{p}$ increases.

In Figs. 2 and 3 we also show the functions $\eta(\tau)$, $\eta_{\max }\left(r_{p}\right)$, and $\tau_{\max }\left(r_{p}\right)$ for several particular thermal plant arrangements. Following Horlock's notation [18], CICIC... BTBT. . . X denotes an arrangement where the solar collector is coupled to a thermal plant with several compressors (C) and intermediate intercoolers (I), several turbines $(\mathrm{T})$ and intermediate reheaters (B), and regeneration (X). 


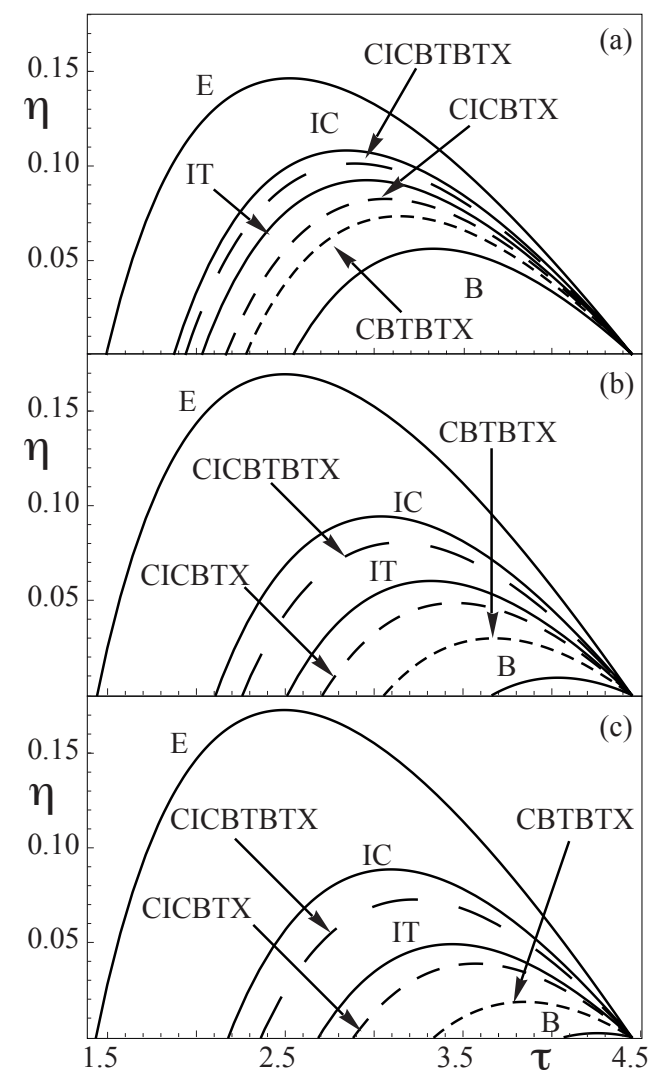

Figure 2: Overall efficiency $\eta$ for different configurations as a function of the temperature ratio and several pressure ratios: (a) $r_{p}=5$; (b) $r_{p}=15$, and (c) $r_{p}=20$.

So, CBTX represents a simple Brayton cycle with regeneration, i.e., the so-called $\mathrm{B}$ arrangement. Because of economical reasons plant configurations over 2 or 3 turbines or compressors are impractical. From Fig. 3(a), we see that at any value of the pressure ratio, the overall optimized efficiency increases in the order B, CBTBTX (one compressor and two turbines), CICBTX (two compressors and one turbine), IT, CICBTBTX (two compressor and two turbines), IC and E. The opposite behavior is found for the optimized temperature ratio in Fig. 3(b).

The most relevant conclusion from Fig. 3(a) is the following: except for $\mathrm{E}, \eta_{\max }$ shows a well defined maximum for small pressure ratio values, while at the same pressure ratios $\tau_{\max }$ shows a minimum. This fact allows an additional optimization of the overall efficiency with respect to $r_{p}[9,10]$.

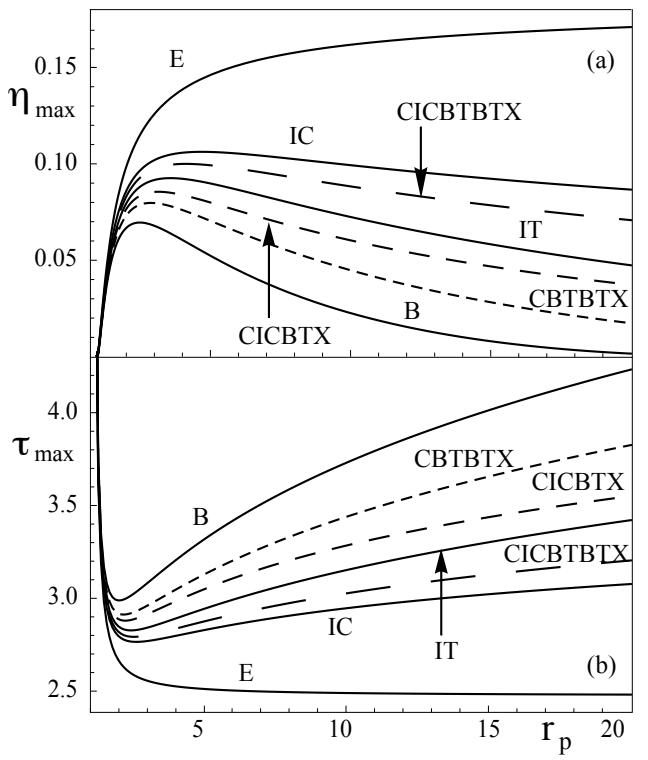

Figure 3: As in Fig. 2 but for the maximum overall efficiency $\eta_{\max }$ (a) and the corresponding temperature ratio $\tau_{\max }$ as functions of the pressure ratio $r_{p}$.(b)

\section{Summary and conclusions}

A thermodynamic analysis for an irreversible solardriven multi-step Brayton heat engine has been developed. Our model could be used as a priori global simulation scheme in order to foresee the overall plant efficiency as a function of a reduced set of parameters with a direct thermodynamic meaning. We have assumed a solar concentrating collector with heat losses accounted through a linear term proportional to an overall effective heat loss coefficient (and checked that all the results reported are insensitive to an explicit radiation losses term). The irreversible thermodynamic cycle model incorporates the possibility of an arbitrary number of turbines $N_{t}$ and compressors $N_{c}$ with the associated reheating and intercooling processes. The overall efficiency of the combined system has been obtained in terms of parameters accounting for both external and internal irreversibility sources. 


\section{Acknowledgements}

We acknowledge financial support from MICINN (Spain) under Grant FIS2010-17147.

\section{References}

[1] SOLGATE. Solar hybrid gas turbine electric power system. Technical Report EUR 21615, European Commission, 2005.

[2] J. Sinai, C. Sugarmen, and U. Fisher. Adaptation and modification of gas turbines for solar energy applications. In Proceedings of GT2005 ASME Turbo Expo 2005, 2005.

[3] A.L. Ávila-Marín. Volumetric receivers in solar thermal power plants with centra receiver system technology: a review. Sol. Energy, 85:891-910, 2011.

[4] R. Chacartegui, J.M. Muñoz de Escalona, D. Sánchez, B. Monje, and T. Sánchez. Alternative cycles based on carbon dioxide for central receiver solar power. Appl. Thermal Eng., 31:872-879, 2011.

[5] S. Sánchez-Orgaz, A. Medina, and A. Calvo Hernández. Thermodynamic model and optimization of a multi-step irreversible Brayton cycle. Energ. Convers. Manage., 51:2134-43, 2010.

[6] Y. Ust. Effects of combined heat transfer on the thermo-economic performance of irreversible solar-driven heat engines. Renew. Energ., 32:2085-2095, 2007.

[7] Y. Zhang, B. Lin, and J. Chen. Optimum performance characteristics off an irrversible solar-driven Brayton heat engine at the maximum overall efficiency. Renew. Energ., 32:856867, 2007.

[8] J.A. Duffie and W.A. Beckman. Solar Engineering of Thermal Processes. John Wiley and Sons, Hoboken, New Jersey, 2006.

[9] S. Sánchez-Orgaz, A. Medina, and A. Calvo Hernández. Recuperative solardriven multi-step gas turbine power plants. Energy Convers. Manage., 67:171-178, 2013.
[10] S. Sánchez-Orgaz, A. Medina, and A. Calvo Hernández. Maximum overall efficiency for a solar-driven gas turbine power plants. Int. J. Energ. Res., DOI:10.1002/er.2967, 2012.

[11] S. Sánchez-Orgaz. Modelization, analysis, and thermodynamic optimization of Brayton multistep power plants. Solar applications. $\mathrm{PhD}$ thesis, Universidad de Salamanca, Spain, 2012.

[12] B. Sunden. High temperature heat exchangers (HTHE). In Proceedings of the Fifth International Conference on Enhanced, Compact and Ultra-Compact Heat Exchangers: Science, Engineering and Technology, Hoboken, NJ, USA, 2005.

[13] M. Kautz and U. Hansen. The externally fired gas turbine (EFGT-cycle) and simulation of the key components. Appl. Energy, 84:795$805,2007$.

[14] A. Romier. Small gas turbine technology. Appl. Thermal Eng., 24:1709-1723, 2004.

[15] EU Project. Coordinator: Microturbo S.A. (France). Research and development of high efficiency components for an intercooled, recuperated CHP gas turbine for combined heat and efficient power. Technical report, Comunidad Europea, Contract No. ENK5-CT-200000070, 2000-2003.

[16] P.E.B. de Mello and D.B. Monteiro. Thermodynamic study of an EFGT (externally fired gas-turbine) cycle with one detailed model for the ceramic heat exchanger. In Proceedings of ECOS 2011 Conference, Novi Sad, Serbia, 2011.

[17] L. Galanti and A.F. Massardo. Micro gas turbine thermodynamic and economic analysis up to $500 \mathrm{kWe}$. Appl. Energy, 88:4795-4802, 2011.

[18] J.H. Horlock. Advanced Gas Turbine Cycles. Pergamon, Oxford, 2003. 